\title{
Review Article \\ Cancer: An Oxidative Crosstalk between Solid Tumor Cells and Cancer Associated Fibroblasts
}

\author{
Alessandro Arcucci, ${ }^{1}$ Maria Rosaria Ruocco, ${ }^{2}$ Giuseppina Granato, ${ }^{1}$ \\ Anna Maria Sacco, ${ }^{1}$ and Stefania Montagnani ${ }^{1}$ \\ ${ }^{1}$ Department of Public Health, University of Naples Federico II, 80131 Naples, Italy \\ ${ }^{2}$ Department of Molecular Medicine and Medical Biotechnology, University of Naples Federico II, 80131 Naples, Italy
}

Correspondence should be addressed to Stefania Montagnani; montagna@unina.it

Received 27 May 2016; Accepted 13 July 2016

Academic Editor: Amit Budhraja

Copyright (C) 2016 Alessandro Arcucci et al. This is an open access article distributed under the Creative Commons Attribution License, which permits unrestricted use, distribution, and reproduction in any medium, provided the original work is properly cited.

Redox balance is associated with the regulation of several cell signalling pathways and functions. In fact, under physiological conditions, cells maintain a balance between oxidant and antioxidant systems, and reactive oxygen species (ROS) can act as second messengers to regulate cell proliferation, cell death, and other physiological processes. Cancer tissues usually contain higher levels of ROS than normal tissues, and this ROS overproduction is associated with tumor development. Neoplastic tissues are very heterogeneous systems, composed of tumor cells and microenvironment that has a critical role in tumor progression. Cancer associated fibroblasts (CAFs) represent the main cell type of tumor microenvironment, and they contribute to tumor growth by undergoing an irreversible activation process. It is known that ROS can be transferred from cancer cells to fibroblasts. In particular, ROS affect the behaviour of CAFs by promoting the conversion of fibroblasts to myofibroblasts that support tumor progression and dissemination. Furthermore, the wrecking of redox homeostasis in cancer cells and tumor microenvironment induces a metabolic reprogramming in tumor cells and cancer associated fibroblasts, giving advantage to cancer growth. This review describes the role of ROS in tumor growth, by focusing on CAFs activation and metabolic interactions between cancer cells and stromal fibroblasts.

\section{Introduction}

Solid tumors are characterized by an abnormal microenvironment regulating tumor progression and providing evasion from cancer therapies [1]. Cancer microenvironment includes blood and lymphatic tumor vessels, extracellular matrix (ECM), and noncancer stromal cells [2] (Figure 1). Blood and lymphatic vessels have a significant role in cancer growth and metastasis $[3,4]$. ECM is formed by interstitial matrix and basement membrane and is very important for the anchorage and migration of cancer cells [2]. Furthermore, ECM contains and stores soluble molecules that can become available for tumor cells. These molecules include chemokines such as CXCL-12, matrix metalloproteinases (MMPs), protease inhibitors, and growth factors such as vascular endothelial growth factor (VEGF) [2]. Noncancer stromal cells are represented by endothelial cells, pericytes, immune cells, cancer associated fibroblasts (CAFs), activated adipocytes, and mesenchymal stem cells (MSCs) [1]. Moreover, all components of tumor microenvironment may vary, depending on the type and location of tumor, thus making each tumor unique [1]. Unlike normal fibroblasts that regulate the turnover of the ECM, control normal tissue homeostasis, and participate in wound healing and senescence, CAFs either reside within the tumor margins or infiltrate the tumor mass and facilitate the tumorigenesis [5]. In particular, the phenotype of CAFs is different from that of normal fibroblasts and is characterized by a more rapid proliferation rate, enhanced collagen production, secretion of growth factors, and ECM modulators [5]. Noncancer stromal cells of almost all solid tumors contain CAFs, whose abundance varies between different cancer types. In particular, breast, prostate, and pancreatic cancers contain high numbers of CAFs; conversely, a reduced number of CAFs is usually present in brain and renal cancers [6]. In breast and pancreatic carcinoma CAFs can represent up 

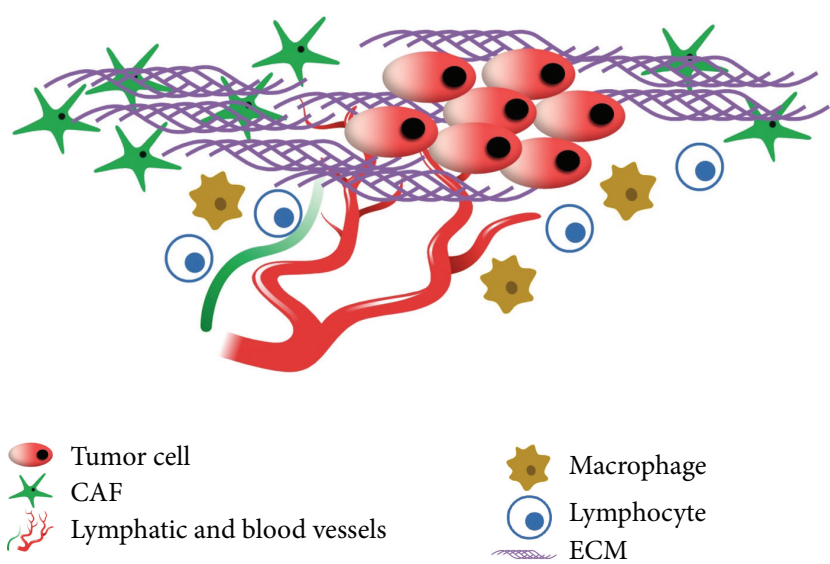

FIGURE 1: Typical components of solid tumors microenvironment.

to $80 \%$ of the tumor mass, as a result of a widespread desmoplasia [7]. Cancer tissues containing a high percentage $(>50 \%)$ of CAFs present increased microvessel density, tumor-associated macrophages, epithelial-mesenchymal transition (EMT), and poor prognosis [7]. CAFs include two cell populations, such as fibroblasts and myofibroblasts, and are represented by nonneoplastic, nonvascular, nonepithelial cells with a fibroblastic phenotype [6]. Several markers are used to detect CAFs in cancer tissues; however, $\alpha$-SMA is the most widely used marker, because there are more myofibroblasts in tumor stroma and $\alpha$-SMA is a specific marker for myofibroblasts [8-10].

Moreover, myofibroblasts represent a cell population of cancer desmoplastic tissues. Indeed, a histological hallmark of cancer tissue is represented by the formation of an abundant tumor stroma formed by ECM and myofibroblasts, inflammatory cells, blood, and lymphatic vessels: this process, named desmoplasia, wrecks the organ's normal architecture [11]. Desmoplasia creates a niche for cancer cells, represents a sign of tumor progression, and can be used as intraoperative prognostic marker [12-14]. In particular, desmoplasia generates mechanical forces that convert fibroblasts and other precursor to myofibroblasts, and it originates a hypoxic and acidic microenvironment that compromises chemotherapeutic treatment [15]. Moreover, hypoxic microenvironment of desmoplastic cancer tissues produces and maintains an oxidative stress condition, because hypoxia is associated with mitochondrial ROS production and glycolytic pathway [16].

However, the whole cancer tissue contains high levels of ROS. In particular, both cancer cells and CAFs produce reactive oxygen species (ROS), such as hydrogen peroxide $\left(\mathrm{H}_{2} \mathrm{O}_{2}\right)$ that induces myofibroblast phenotype in CAFs and cancer alterations in epithelial cells: these processes influence the behaviour of neighboring CAFs and cancer cells $[16,17]$.

Therefore, this review describes the role of ROS in CAFs activation and in the regulation of tumor-stroma interaction.

\section{ROS in Cancer Cells}

ROS are a group of chemical species, unstable and reactive derivatives of oxygen. They are represented by electrophilic and highly reactive molecules such as radical forms represented mainly by superoxide anion $\left(\mathrm{O}_{2}{ }^{--}\right)$, hydroxyl $\left(\mathrm{OH}^{*}\right)$, carbon dioxide anion $\left(\mathrm{CO}_{2}{ }^{--}\right)$, and nonradical forms, such as $\mathrm{H}_{2} \mathrm{O}_{2}$ [16]. ROS sources can be endogenous or exogenous [18]. Endogenous ROS are produced mainly by mitochondria during oxidative phosphorylation (OXPHOS) process, NADPH oxidase enzymes (NOX), and cytochromes: the ROS production begins with the transfer of one electron to molecular oxygen $\left(\mathrm{O}_{2}\right)$ to form $\mathrm{O}_{2}{ }^{--}$which can dismutate into $\mathrm{H}_{2} \mathrm{O}_{2}$ spontaneously at low $\mathrm{pH}$ or through enzymatic activity of the superoxide dismutase (SOD) enzyme [16, 19]. Moreover, $\mathrm{H}_{2} \mathrm{O}_{2}$ can be transformed, through the Fenton reaction, to highly reactive and toxic $\mathrm{OH}^{*}[16,19]$. Exogenous ROS sources are represented by pollutants, tobacco, smoke, drugs, xenobiotics, and radiations $[18,20]$.

ROS homeostasis depends on the balance between ROS production and enzymatic and nonenzymatic antioxidant systems; indeed, the wrecking of this equilibrium leads to oxidative stress, which can contribute to tumor development [21]. In particular, oxidative stress can alter the cellular physiological functioning of the redox-sensitive mechanisms, without inducing an irreversible oxidative damage or general oxidative cell condition [21]. Moreover, the typical hypoxic tumor microenvironment regulates cancer invasiveness through mitochondrial redox signalling [22]. Hypoxia induces the mitochondrial production of $\mathrm{O}_{2}{ }^{--}$that is transformed to $\mathrm{H}_{2} \mathrm{O}_{2}$ by SOD2 enzymatic activity [21]. $\mathrm{H}_{2} \mathrm{O}_{2}$, one of the most stable and permeable ROS, can traverse the mitochondrial and cell membranes and it represents the main ROS in the regulation of signalling transduction pathways, affecting the behaviour of cancer and stromal cells $[16,19,22]$. Furthermore, aquaporin- 8 that is a transmembrane protein localized in the plasma and inner mitochondrial membrane facilitates the diffusion of $\mathrm{H}_{2} \mathrm{O}_{2}$ across cellular membranes [23]. In a hepatocellular carcinoma cell line the silencing of aquaporin-8 gene impairs $\mathrm{H}_{2} \mathrm{O}_{2}$ mitochondrial releases, increases mitochondrial ROS, and induces a ROS-dependent mitochondrial depolarization as well as a loss of cell viability [24]. Moreover, there are several works showing and explaining the positive function of aquaporin- 8 in carcinogenesis [25-28]. In conclusion, the perturbation of cellular redox homeostasis can represent one of the main factors inducing and maintaining tumor phenotype in cancer tissues.

\section{ROS and Cancer Associated Fibroblasts Activation}

The pivotal role of microenvironment in tumor development, progression, and metastasis is known. In particular, the most abundant cell population in the stroma of solid tumors is represented by CAFs [29]. CAFs play a key role in tumorigenesis, tumor support, and progression and are characterized by a heterogeneous cell origin. Although CAFs can derive from epithelial or endothelial cells through an epithelial or endothelial mesenchymal transition (EMT/EndMT) [30-32], EMT/EndMT cannot be considered the only origin of CAFs. CAFs can also derive from hematopoietic stem cells [33], pericytes, and adipocytes [34], although tumor stroma resident fibroblasts are one of the main sources of CAFs $[35,36]$. 
However, most of CAFs are phenotypically and epigenetically different with respect to normal dermal fibroblasts. Indeed, CAFs can be considered a cell population in a permanent activated state, sharing similarities with fibroblasts activated during wound healing [37]. The activated CAFs promote tumorigenesis and cancer development through secretion of growth factors, cytokines, and paracrine interactions [6]. In breast cancer over $80 \%$ of CAFs are myofibroblasts that, contrary to wound healing process, are constitutively activated and neither revert to a normal phenotype nor undergo apoptosis [38]. Breast CAFs show high expression of $\alpha$-SMA, p53, podoplanin, CD10, fibroblast activation protein (FAP), matrix metalloproteinases (MMPs), tenascin-C, and platelet-derived growth factor receptor (PDGFR $\alpha / \beta)$ and lose caveolin-1 (Cav-1) expression [39]. Other markers such as fibroblast-specific protein 1 (FSP1), neuron-glial antigen 2 (NG2), and prolyl 4-hydroxylase can be useful to identify CAFs subtypes in carcinomas [6]. The irreversible activation of CAFs is due to signalling pathways driven by several factors produced mainly by tumor cells and autocrine loops $[16,37]$. Different studies showed that ROS have a pivotal role in the process of fibroblast activation. TGF- $\beta 1$, involved in fibroblast-to-myofibroblast switch, induces an increase of fibroblasts ROS that mediate the expression of $\alpha$-SMA [40]. One of the main sources of this ROS boost seems to be represented by NOX associated with fibroblasts membranes [41, 42], and in particular many studies have observed that NOX4 represents the main target of TGF- $\beta 1$ in the process of fibroblasts activation [43-45]. Furthermore, TGF$\beta 1$ activates a mitochondrial ROS production that is also required for Nox4 transcription. Hence, NOX4 expression, amplifying the ROS mitochondrial burst, can prolong TGF$\beta 1$ signalling [46]. In addition, the unregulated mitochondrial ROS production is associated with fibrosis evolution as well as cancer progression and invasion [16, 47]. In fibroblasts, a genetic dysfunction of mitochondrial complex I produces an oxidative stress triggering fibroblasts activation; under these conditions, myofibroblasts promote a proinvasive phenotype of human melanoma cells through the secretion of cytokines [48]. Oxidative status of tumor stroma can affect cancer cells dissemination. Indeed, it has been observed that, in a model of mammary carcinogenesis, $j u n D^{-1-}$ stromal fibroblasts increase the metastatic potential of neoplastic cells [49]. JunD, a member of activator protein-1, positively regulates some antioxidant genes [50]. Furthermore in a jun $D^{-/-}$fibroblasts system the activation of fibroblasts into myofibroblasts was linked to an increase of oxidative status [49]. Conversely, the decrease of oxidative stress in tumor stroma can reduce cancer mass and revert its metastatic potential. Indeed, it has been shown that Cav-1 and SOD2 in CAFs can act as oncosuppressors [51]. Moreover, in prostatic hyperplasia and prostate cancer, the upregulation of antioxidant selenoenzymes, such as glutathione peroxidase 3 and thioredoxin reductase I, inhibits the differentiation of fibroblasts into myofibroblasts through the reduction of ROS levels produced by NOX4 [52].

ROS can affect the differentiation and the tumorigenic potential of a heterogeneous cell population, such as
CAFs, through different mechanisms. Indeed, in a PDGFR- $\beta$ fibroblast subtype ROS mediate PDGF signalling through phosphatases inactivation [53]. It has been shown that the reversible inhibition of phosphatases by PDGF-induced ROS is involved in the fibroblast proliferation and migration [54]. Many studies have evidenced NOX as the main source of ROS induced upon growth factors signalling [55-57], although also mitochondrial ROS can contribute to the oxidation and inhibition of protein phosphatases [58]. Furthermore, ROS modulated Cav-1 expression is closely linked to the role of CAFs as tumor feeder and to biochemical remodelling of the microenvironment [16]. Hence, it is possible that different ROS species can evoke specific effects on $\mathrm{Cav}_{-1}{ }^{+} \mathrm{CAFs}$. Indeed, it has been demonstrated that in lung cancer cells Cav-1 is differentially modulated by various ROS [59]. In the complex, ROS represent main factors in the fibroblast activation process as well as in CAFs behaviour, although further studies will be necessary to better understand signalling pathways activated by ROS.

\section{ROS and Metabolic Interaction between Stromal Fibroblasts and Cancer Cells}

It is known that cancer tissues produce high levels of ROS that can derive from mitochondrial dysfunction, upregulation of NOX1 and NOX4, and alterations of antioxidant enzymes [16]. In particular, the mitochondrial dysfunction due to impairment of mitochondrial electron transport chain produces a considerable $\mathrm{O}_{2}{ }^{--}$production [16]. The mitochondrial dysfunction is usually associated with a switch to aerobic glycolysis, known as Warburg effect that is an early step of carcinogenesis, and can occur before the appearance of a hypoxic tumor environment [60]. In fact, lung cancer cells grow in the presence of oxygen and utilize glycolysis to produce ATP [60]. Glycolysis produces lower amount of ATP with respect to OXPHOS, but the glycolytic choice offers to cancer cells a fast ATP production and glycolytic intermediates that contribute to the growth of cancer cells $[19,60]$. During glycolysis the transformation of pyruvate into lactate leads to a deficit of pyruvate, the primary fuel supporting Krebs cycle: this process deprives cancer cells of some Krebs cycle intermediates with antioxidant activity [16]. However, hypoxia is the main factor inducing glycolytic switch in cancer cells: it is associated with ROS production by mitochondrial complex III and induces the activation of HIF-1 $\alpha$ that contributes to glycolytic pathway [61]. In particular, HIF-1 $\alpha$ regulates the switch from OXPHOS to glycolysis through the induction of enzymes involved in the glycolysis and overexpression of glucose transporters GLUT1 and GLUT3 [62]. Moreover, HIF-1 $\alpha$ can be activated also by PI3K/AKT/mTOR signalling pathway, and HIF-1 $\alpha$ levels can be correlated with the severity of many tumors $[60,63]$. Therefore, in cancer cells both Warburg effect and mitochondrial malfunctioning induce an increase of lactate and ROS levels and a decrease of antioxidant molecules [16].

It is known that cancer cells produce large amounts of $\mathrm{H}_{2} \mathrm{O}_{2}$ without exogenous stimulation [64]. $\mathrm{H}_{2} \mathrm{O}_{2}$ produced and secreted by cancer cells "fertilizes" the tumor microenvironment and induces oxidative stress in CAFs. 
Martinez-Outschoorn studied $\mathrm{H}_{2} \mathrm{O}_{2}$ stromal effect, by coculturing a breast cancer cell line with immortalized fibroblasts $[65,66]$. In particular, the transfer of oxidative stress from cancer cells to CAFs is associated with the reduction of mitochondrial function and the increase of both glucose uptake and ROS levels in CAFs [65]. On the other hand, cocultured cancer cells show significant increase of mitochondrial activity and decrease of both GLUT1 expression and glucose uptake. This process is interrupted if catalase is added to the cell culture media [65]. Furthermore, fibroblasts cocultured with breast cancer cells acquire a CAFs phenotype characterized by Cav-1 downregulation, increased expression of myofibroblast markers, extracellular matrix proteins, and constitutive activation of TGF- $\beta / \mathrm{Smad} 2$ signalling pathway [66]. In particular, TGF- $\beta$ induces differentiation of prostate CAFs to myofibroblasts by triggering NOX4 upregulation and elevated ROS production [52]. Moreover, TGF- $\beta$ triggers in fibroblasts increased oxidative stress, autophagy/mitophagy, aerobic glycolysis, and downregulation of Cav-1: these alterations can extend to surrounding fibroblasts and support cancer cell growth [67]. Loss of stromal Cav-1 may be used as biomarker for cancer aggressiveness $[68,69]$. Cav-1 is a structural component of caveolae that are flask-shaped invaginations of the plasma membrane enriched in sphingolipids and cholesterol and are involved in several cellular functions, such as vesicular transport, cholesterol homeostasis, and signal transduction pathways $[70,71]$. In particular, Cav-1 can regulate many transduction pathways by interacting with several signalling proteins localized in lipid rafts and caveolar membranes [72]. A recent work showed that Cav-1 is a negative regulator of ROS produced by NOX enzymes and this is realised by several mechanisms such as enzyme binding and inhibition of endogenous Nox gene expression [73]. In particular Cav1 can inhibit ROS production from NOX2 and NOX5 by direct enzyme binding, via allosteric regulation. Moreover, in addition to this posttranslational regulation, Cav-1 represses NOX2 and NOX4 gene expression and protein synthesis through inhibition of the NF- $k$ B pathway [73]. ROS produced by cancer cells induce loss of Cav-1 in stromal cells, driving glycolysis switch and lactate excretion. This process is associated with HIF-1 $\alpha$ stabilization and upregulation of the monocarboxylate transporter MCT4, which is a biosensor of oxidative stress in CAFs [74, 75]. On the other hand, breast cancer cells, cocultured with fibroblasts, showed the upregulation of MCT1, the transporter involved in lactate uptake [74]. Hence, cancer cells induce in CAFs oxidative stress and a glycolysis switch associated with Cav-1 downregulation. In particular, loss of Cav-1 could be a marker of glycolysis in CAFs that increase in cancer cells OXPHOS rate, ATP production, and proliferation, by supplying tumor cells with lactate in a paracrine manner [76]. Furthermore, 3-hydroxybutyrate and L-lactate, end-products of aerobic glycolysis, feed tumor growth and metastasis in a human tumor xenograft experimental system [77]. In particular, 3hydroxybutyrate induces an increase in tumor volume without any increase in angiogenesis, whereas L-lactate stimulates significantly the formation of metastases [77]. This paracrine metabolic crosstalk between CAFs and cancer cells could also represent a mechanism that confers drug-resistance during antiangiogenic therapy, by reducing the dependence of cancer cells on a vascular blood supply [78, 79]. Finally, it is important to note that standard chemotherapies induce in dermal fibroblast cell lines an activated CAFs phenotype, characterized by $\alpha$-SMA expression, glycolytic switch, ROS production, senescence, autophagy, and increased secretion of interleukin 6. Hence, this catabolic and inflammatory microenvironment represents an ideal niche to sustain carcinogenesis [80].

Therefore, these observations lead to considering ROS as a bridge between tumor cells and CAFs, thus contributing to cancer development, progression, and metastasis.

\section{Conclusions}

Cancer tissues represent a network formed by cancer cells and microenvironment, where the close interactions between tumor cells and CAFs contribute to cancer growth and progression. Tumors show higher ROS levels than normal tissues, and ROS alterations have significant implications in tumor growth and metastasis. ROS induce tumorigenesis by affecting the behaviour of both tumor cells and CAFs and regulating their metabolic interactions. On the other hand, it is important to note that ROS are also involved in the activation of natural defences against the appearance and dissemination of cancer cells [20]. In particular, oxidative stress can limit distant metastasis of melanomas in vivo [81]. Moreover, successfully metastasizing melanomas undergo reversible metabolic changes, raising their capacity to resist oxidative stress. These metabolic changes include increased dependence upon NADPH-generating enzymes in the folate pathway, which is closely linked to GSH regeneration [81]. However, when ROS levels in tumor tissues become extremely high, they can induce cancer cell death. Indeed, the cytotoxicity of many chemotherapeutic drugs is mediated by a further increase of ROS levels in tumor tissues [82]. However, upon drug treatment some cancer cells can undergo a process of "redox resetting" associated with a new redox balance characterized by higher levels of ROS and stronger antioxidant systems. "Redox resetting" enables tumors to become resistant to anticancer drugs: it is possible to bypass this problem by combining drugs that generate ROS with compounds that downregulate the cellular antioxidant capacity [82]. Furthermore, recent works have demonstrated that CAFs not only play an important role in tumorigenesis but also contribute to chemotherapy resistance [83]. Hence, future therapeutic strategies will require the targeting of both cancer cells and stromal fibroblasts.

\section{Competing Interests}

The authors declare that there are no competing interests regarding the publication of this paper.

\section{Acknowledgments}

This work was supported by a grant from Regione Campania (Italy): GR-2009-1580434. 


\section{References}

[1] V. Gkretsi, A. Stylianou, P. Papageorgis, C. Polydorou, and T. Stylianopoulos, "Remodeling components of the tumor microenvironment to enhance cancer therapy," Frontiers in Oncology, vol. 5, article 214, 2015.

[2] A. Alphonso and S. K. Alahari, "Stromal cells and integrins: conforming to the needs of the tumor microenvironment," Neoplasia, vol. 11, no. 12, pp. 1264-1271, 2009.

[3] S. H. Lee, D. Jeong, Y. Han, and M. J. Baek, "Pivotal role of vascular endothelial growth factor pathway in tumor angiogenesis," Annals of Surgical Treatment and Research, vol. 89, no. 1, pp. 1-8, 2015.

[4] X. Guan, "Cancer metastases: challenges and opportunities," Acta Pharmaceutica Sinica B, vol. 5, no. 5, pp. 402-418, 2015.

[5] S. Madar, I. Goldstein, and V. Rotter, "Cancer associated fibroblasts'-more than meets the eye," Trends in Molecular Medicine, vol. 19, no. 8, pp. 447-453, 2013.

[6] D. Öhlund, E. Elyada, and D. Tuveson, "Fibroblast heterogeneity in the cancer wound," The Journal of Experimental Medicine, vol. 211, no. 8, pp. 1503-1523, 2014.

[7] P. Gascard and T. D. Tlsty, "Carcinoma-associated fibroblasts: orchestrating the composition of malignancy," Genes \& Development, vol. 30, no. 9, pp. 1002-1019, 2016.

[8] A.-P. Sappino, O. Skalli, B. Jackson, W. Schürch, and G. Gabbiani, "Smooth-muscle differentiation in stromal cells of malignant and non-malignant breast tissues," International Journal of Cancer, vol. 41, no. 5, pp. 707-712, 1988.

[9] A. Orimo and R. A. Weinberg, "Heterogeneity of stromal fibroblasts in tumors," Cancer Biology \& Therapy, vol. 6, no. 4, pp. 618-619, 2007.

[10] B. Hinz, S. H. Phan, V. J. Thannickal et al., "Recent developments in myofibroblast biology: paradigms for connective tissue remodeling," The American Journal of Pathology, vol. 180, no. 4, pp. 1340-1355, 2012.

[11] A. Neesse, P. Michl, K. K. Frese et al., "Stromal biology and therapy in pancreatic cancer," Gut, vol. 60, no. 6, pp. 861-868, 2011.

[12] Y. A. DeClerck, "Desmoplasia: a response or a niche?" Cancer Discovery, vol. 2, no. 9, pp. 772-774, 2012.

[13] C. Scheuba, K. Kaserer, K. Kaczirek, R. Asari, and B. Niederle, "Desmoplastic stromal reaction in medullary thyroid canceran intraoperative 'marker' for lymph node metastases," World Journal of Surgery, vol. 30, no. 5, pp. 853-859, 2006.

[14] O. Koperek, R. Asari, B. Niederle, and K. Kaserer, "Desmoplastic stromal reaction in papillary thyroid microcarcinoma," Histopathology, vol. 58, no. 6, pp. 919-924, 2011.

[15] R. K. Jain, J. D. Martin, and T. Stylianopoulos, "The role of mechanical forces in tumor growth and therapy," Annual Review of Biomedical Engineering, vol. 16, pp. 321-346, 2014.

[16] A. Costa, A. Scholer-Dahirel, and F. Mechta-Grigoriou, "The role of reactive oxygen species and metabolism on cancer cells and their microenvironment," Seminars in Cancer Biology, vol. 25, pp. 23-32, 2014.

[17] A. Jezierska-Drutel, S. A. Rosenzweig, and C. A. Neumann, "Role of oxidative stress and the microenvironment in breast cancer development and progression," Advances in Cancer Research, vol. 119, pp. 107-125, 2013.

[18] S. Prasad, S. C. Gupta, and A. K. Tyagi, "Reactive oxygen species (ROS) and cancer: role of antioxidative nutraceuticals," Cancer Letters, vol. 3835, no. 16, pp. 30202-30206, 2016.
[19] E. Gammella, S. Recalcati, and G. Cairo, "Dual role of ROS as signal and stress agents: iron tips the balance in favor of toxic effects," Oxidative Medicine and Cellular Longevity, vol. 2016, Article ID 8629024, 9 pages, 2016.

[20] F. Albano, A. Arcucci, G. Granato et al., "Markers of mitochondrial dysfunction during the diclofenac-induced apoptosis in melanoma cell lines," Biochimie, vol. 95, no. 4, pp. 934-945, 2013.

[21] F. Hecht, C. F. Pessoa, L. B. Gentile, D. Rosenthal, D. P. Carvalho, and R. S. Fortunato, "The role of oxidative stress on breast cancer development and therapy," Tumor Biology, vol. 37, no. 4, pp. 4281-4291, 2016.

[22] L. Enns and W. Ladiges, "Mitochondrial redox signaling and cancer invasiveness," Journal of Bioenergetics and Biomembranes, vol. 44, no. 6, pp. 635-638, 2012.

[23] G. P. Bienert, A. L. B. Møller, K. A. Kristiansen et al., "Specific aquaporins facilitate the diffusion of hydrogen peroxide across membranes," The Journal of Biological Chemistry, vol. 282, no. 2, pp. 1183-1192, 2007.

[24] M. J. Marchissio, D. E. A. Francés, C. E. Carnovale, and R. A. Marinelli, "Mitochondrial aquaporin-8 knockdown in human hepatoma HepG2 cells causes ROS-induced mitochondrial depolarization and loss of viability," Toxicology and Applied Pharmacology, vol. 264, no. 2, pp. 246-254, 2012.

[25] H. Chang, Y.-H. Shi, T.-K. Talaf, and C. Lin, "Aquaporin-8 mediates human esophageal cancer Eca-109 cell migration via the EGFR-Erk1/2 pathway," International Journal of Clinical and Experimental Pathology, vol. 7, no. 11, pp. 7663-7671, 2014.

[26] H. Chang, Y. Shi, T. Tuokan, R. Chen, and X. Wang, "Expression of aquaporin 8 and phosphorylation of Erk1/2 in cervical epithelial carcinogenesis: correlation with clinicopathological parameters," International Journal of Clinical and Experimental Pathology, vol. 7, no. 7, pp. 3928-3937, 2014.

[27] S.-J. Zhu, K.-J. Wang, S.-W. Gan, J. Xu, S.-Y. Xu, and S.-Q. Sun, "Expression of aquaporin8 in human astrocytomas: correlation with pathologic grade," Biochemical and Biophysical Research Communications, vol. 440, no. 1, pp. 168-172, 2013.

[28] F. Vieceli Dalla Sega, L. Zambonin, D. Fiorentini et al., "Specific aquaporins facilitate Nox-produced hydrogen peroxide transport through plasma membrane in leukaemia cells," Biochimica et Biophysica Acta (BBA)-Molecular Cell Research, vol. 1843, no. 4, pp. 806-814, 2014.

[29] K. Pietras and A. Östman, "Hallmarks of cancer: interactions with the tumor stroma," Experimental Cell Research, vol. 316, no. 8, pp. 1324-1331, 2010.

[30] E. S. Radisky and D. C. Radisky, "Stromal induction of breast cancer: inflammation and invasion," Reviews in Endocrine and Metabolic Disorders, vol. 8, no. 3, pp. 279-287, 2007.

[31] J. Zavadil, J. Haley, R. Kalluri, S. K. Muthuswamy, and E. Thompson, "Epithelial-mesenchymal transition," Cancer Research, vol. 68, no. 23, pp. 9574-9577, 2008.

[32] E. M. Zeisberg, S. Potenta, L. Xie, M. Zeisberg, and R. Kalluri, "Discovery of endothelial to mesenchymal transition as a source for carcinoma-associated fibroblasts," Cancer Research, vol. 67, no. 21, pp. 10123-10128, 2007.

[33] L. T. McDonald, D. L. Russell, R. R. Kelly et al., "Hematopoietic stem cell-derived cancer-associated fibroblasts are novel contributors to the pro-tumorigenic microenvironment," Neoplasia, vol. 17, no. 5, pp. 434-448, 2015.

[34] B. Dirat, L. Bochet, M. Dabek et al., "Cancer-associated adipocytes exhibit an activated phenotype and contribute to breast cancer invasion," Cancer Research, vol. 71, no. 7, pp. 24552465, 2011. 
[35] Y. Kojima, A. Acar, E. N. Eaton et al., "Autocrine TGF- $\beta$ and stromal cell-derived factor-1 (SDF-1) signaling drives the evolution of tumor-promoting mammary stromal myofibroblasts," Proceedings of the National Academy of Sciences of the United States of America, vol. 107, no. 46, pp. 20009-20014, 2010.

[36] L. Mueller, F. A. Goumas, M. Affeldt et al., "Stromal fibroblasts in colorectal liver metastases originate from resident fibroblasts and generate an inflammatory microenvironment," The American Journal of Pathology, vol. 171, no. 5, pp. 1608-1618, 2007.

[37] M. Augsten, "Cancer-associated fibroblasts as another polarized cell type of the tumor microenvironment," Frontiers in Oncology, vol. 4, article 62, Article ID Article 62, 2014.

[38] K. Shiga, M. Hara, T. Nagasaki, T. Sato, H. Takahashi, and H. Takeyama, "Cancer-associated fibroblasts: their characteristics and their roles in tumor growth," Cancers, vol. 7, no. 4, pp. 24432458, 2015.

[39] R. J. Buchsbaum and S. Y. Oh, "Breast cancer-associated fibroblasts: where we are and where we need to go," Cancers, vol. 8, no. 2, article 19, 2016.

[40] V. Catalano, A. Turdo, S. Di Franco, F. Dieli, M. Todaro, and G. Stassi, "Tumor and its microenvironment: a synergistic interplay," Seminars in Cancer Biology, vol. 23, no. 6, pp. 522532, 2013.

[41] L. Alili, M. Sack, K. Puschmann, and P. Brenneisen, "Fibroblastto-myofibroblast switch is mediated by $\mathrm{NAD}(\mathrm{P}) \mathrm{H}$ oxidase generated reactive oxygen species," Bioscience Reports, vol. 34, no. 1, Article ID e00089, 2014.

[42] E. Crosas-Molist, E. Bertran, and I. Fabregat, "Cross-talk between TGF- $\beta$ and NADPH oxidases during liver fibrosis and hepatocarcinogenesis," Current Pharmaceutical Design, vol. 21, no. 41, pp. 5964-5976, 2015.

[43] L. Hecker, R. Vittal, T. Jones et al., "NADPH oxidase-4 mediates myofibroblast activation and fibrogenic responses to lung injury," Nature Medicine, vol. 15, no. 9, pp. 1077-1081, 2009.

[44] N. Amara, D. Goven, F. Prost, R. Muloway, B. Crestani, and J. Boczkowski, "NOX4/NADPH oxidase expression is increased in pulmonary fibroblasts from patients with idiopathic pulmonary fibrosis and mediates TGF $\beta 1$-induced fibroblast differentiation into myofibroblasts," Thorax, vol. 65, no. 8, pp. 733738, 2010.

[45] I. Cucoranu, R. Clempus, A. Dikalova et al., "NAD(P)H oxidase 4 mediates transforming growth factor- $\beta 1$-induced differentiation of cardiac fibroblasts into myofibroblasts," Circulation Research, vol. 97, no. 9, pp. 900-907, 2005.

[46] M. Jain, S. Rivera, E. A. Monclus et al., "Mitochondrial reactive oxygen species regulate transforming growth factor- $\beta$ signaling," The Journal of Biological Chemistry, vol. 288, no. 2, pp. 770 777, 2013.

[47] R. Samarakoon, J. M. Overstreet, and P. J. Higgins, “TGF- $\beta$ signaling in tissue fibrosis: redox controls, target genes and therapeutic opportunities," Cellular Signalling, vol. 25, no. 1, pp. 264-268, 2013.

[48] M. L. Taddei, E. Giannoni, G. Raugei et al., "Mitochondrial oxidative stress due to complex I dysfunction promotes fibroblast activation and melanoma cell invasiveness," Journal of Signal Transduction, vol. 2012, Article ID 684592, 10 pages, 2012.

[49] A. Toullec, D. Gerald, G. Despouy et al., "Oxidative stress promotes myofibroblast differentiation and tumour spreading," EMBO Molecular Medicine, vol. 2, no. 6, pp. 211-230, 2010.

[50] D. Gerald, E. Berra, Y. M. Frapart et al., "JunD reduces tumor angiogenesis by protecting cells from oxidative stress," Cell, vol. 118, no. 6, pp. 781-794, 2004.
[51] C. Trimmer, F. Sotgia, D. Whitaker-Menezes et al., "Caveolin1 and mitochondrial SOD2 (MnSOD) function as tumor suppressors in the stromal microenvironment: a new genetically tractable model for human cancer associated fibroblasts," Cancer Biology and Therapy, vol. 11, no. 4, pp. 383-394, 2011.

[52] N. Sampson, R. Koziel, C. Zenzmaier et al., "ROS signaling by NOX4 drives fibroblast-to-myofibroblast differentiation in the diseased prostatic stroma," Molecular Endocrinology, vol. 25, no. 3, pp. 503-515, 2011.

[53] D. Xu, I. I. Rovira, and T. Finkel, "Oxidants painting the cysteine chapel: redox regulation of PTPs," Developmental Cell, vol. 2, no. 3, pp. 251-252, 2002.

[54] T.-C. Meng, T. Fukada, and N. K. Tonks, "Reversible oxidation and inactivation of protein tyrosine phosphatases in vivo," Molecular Cell, vol. 9, no. 2, pp. 387-399, 2002.

[55] P. Sharma, R. Chakraborty, L. Wang et al., "Redox regulation of interleukin-4 signaling," Immunity, vol. 29, no. 4, pp. 551-564, 2008.

[56] R. F. Wu, Y. C. Xu, Z. Ma, F. E. Nwariaku, G. A. Sarosi Jr., and L. S. Terada, "Subcellular targeting of oxidants during endothelial cell migration," The Journal of Cell Biology, vol. 171, no. 5, pp. 893-904, 2005.

[57] K. Chen, M. T. Kirber, H. Xiao, Y. Yang, and J. F. Keaney Jr., "Regulation of ROS signal transduction by NADPH oxidase 4 localization," The Journal of Cell Biology, vol. 181, no. 7, pp. 11291139, 2008.

[58] J. Frijhoff, M. Dagnell, M. Augsten, E. Beltrami, M. Giorgio, and A. Östman, "The mitochondrial reactive oxygen species regulator p66Shc controls PDGF-induced signaling and migration through protein tyrosine phosphatase oxidation," Free Radical Biology and Medicine, vol. 68, pp. 268-277, 2014.

[59] S. Luanpitpong, S. J. Talbott, Y. Rojanasakul et al., "Regulation of lung cancer cell migration and invasion by reactive oxygen species and caveolin-1," The Journal of Biological Chemistry, vol. 285, no. 50, pp. 38832-38840, 2010.

[60] L. Yu, X. Chen, L. Wang, and S. Chen, "The sweet trap in tumors: aerobic glycolysis and potential targets for therapy," Oncotarget, vol. 7, no. 25, pp. 38908-38926, 2016.

[61] N. S. Chandel, D. S. McClintock, C. E. Feliciano et al., "Reactive oxygen species generated at mitochondrial complex III stabilize hypoxia-inducible factor- $1 \alpha$ during hypoxia: a mechanism of $\mathrm{O}_{2}$ sensing," The Journal of Biological Chemistry, vol. 275, no. 33, pp. 25130-25138, 2000.

[62] L. Zhao, Y. Mao, Y. Zhao, Y. Cao, and X. Chen, "Role of multifaceted regulators in cancer glucose metabolism and their clinical significance," Oncotarget, vol. 7, no. 21, pp. 31572-31585, 2016.

[63] G. L. Semenza, "HIF-1 mediates metabolic responses to intratumoral hypoxia and oncogenic mutations," The Journal of Clinical Investigation, vol. 123, no. 9, pp. 3664-3671, 2013.

[64] T. P. Szatrowski and C. F. Nathan, "Production of large amounts of hydrogen peroxide by human tumor cells," Cancer Research, vol. 51, no. 3, pp. 794-798, 1991.

[65] U. E. Martinez-Outschoorn, Z. Lin, C. Trimmer et al., "Cancer cells metabolically 'fertilize' the tumor microenvironment with hydrogen peroxide, driving the Warburg effect: Implications for PET imaging of human tumors," Cell Cycle, vol. 10, no. 15, pp. 2504-2520, 2011.

[66] U. E. Martinez-Outschoorn, S. Pavlides, D. Whitaker-Menezes et al., "Tumor cells induce the cancer associated fibroblast phenotype via caveolin-1 degradation: implications for breast 
cancer and DCIS therapy with autophagy inhibitors," Cell Cycle, vol. 9, no. 12, pp. 2423-2433, 2014.

[67] C. Guido, D. Whitaker-Menezes, C. Capparelli et al., "Metabolic reprogramming of cancer-associated fibroblasts by TGF$\beta$ drives tumor growth: connecting TGF- $\beta$ signaling with 'Warburg- like' cancer metabolism and L-lactate production," Cell Cycle, vol. 11, no. 16, pp. 3019-3035, 2012.

[68] T. Shan, H. Lu, H. Ji et al., "Loss of stromal caveolin-1 expression: a novel tumor microenvironment biomarker that can predict poor clinical outcomes for pancreatic cancer," PLOS ONE, vol. 9, no. 6, Article ID e97239, 2014.

[69] A. K. Witkiewicz, A. Dasgupta, F. Sotgia et al., "An absence of stromal caveolin-1 expression predicts early tumor recurrence and poor clinical outcome in human breast cancers," The American Journal of Pathology, vol. 174, no. 6, pp. 2023-2034, 2009.

[70] P. U. Le, G. Guay, Y. Altschuler, and I. R. Nabi, "Caveolin-1 is a negative regulator of caveolae-mediated endocytosis to the endoplasmic reticulum," The Journal of Biological Chemistry, vol. 277, no. 5, pp. 3371-3379, 2002.

[71] T.-K. Ha, N.-G. Her, M.-G. Lee et al., "Caveolin-1 increases aerobic glycolysis in colorectal cancers by stimulating HMGA1mediated GLUT3 transcription," Cancer Research, vol. 72, no. 16, pp. 4097-4109, 2012.

[72] R. Gupta, C. Toufaily, and B. Annabi, "Caveolin and cavin family members: dual roles in cancer," Biochimie, vol. 107, pp. 188-202, 2014.

[73] F. Chen, S. Barman, Y. Yu et al., "Caveolin-1 is a negative regulator of NADPH oxidase-derived reactive oxygen species," Free Radical Biology \& Medicine, vol. 73, pp. 201-213, 2014.

[74] D. Whitaker-Menezes, U. E. Martinez-Outschoorn, Z. Lin et al., "Evidence for a stromal-epithelial 'lactate shuttle' in human tumors: MCT4 is a marker of oxidative stress in cancerassociated fibroblasts," Cell Cycle, vol. 10, no. 11, pp. 1772-1783, 2011.

[75] U. E. Martinez-Outschoorn, F. Sotgia, and M. P. Lisanti, “Tumor microenvironment and metabolic synergy in breast cancers: critical importance of mitochondrial fuels and function," Seminars in Oncology, vol. 41, no. 2, pp. 195-216, 2014.

[76] S. Pavlides, D. Whitaker-Menezes, R. Castello-Cros et al., "The reverse Warburg effect: aerobic glycolysis in cancer associated fibroblasts and the tumor stroma," Cell Cycle, vol. 8, no. 23, pp. 3984-4001, 2009.

[77] G. Bonuccelli, A. Tsirigos, D. Whitaker-Menezes et al., "Ketones and lactate 'fuel' tumor growth and metastasis: evidence that epithelial cancer cells use oxidative mitochondrial metabolism," Cell Cycle, vol. 9, no. 17, pp. 3506-3514, 2010.

[78] U. E. Martinez-Outschoorn, R. G. Pestell, A. Howell et al., "Energy transfer in 'parasitic' cancer metabolism: mitochondria are the powerhouse and Achilles' heel of tumor cells," Cell Cycle, vol. 10, no. 24, pp. 4208-4216, 2011.

[79] G. Migneco, D. Whitaker-Menezes, B. Chiavarina et al., "Glycolytic cancer associated fibroblasts promote breast cancer tumor growth, without a measurable increase in angiogenesis: evidence for stromal-epithelial metabolic coupling," Cell Cycle, vol. 9, no. 12, pp. 2412-2422, 2010.

[80] M. Peiris-Pagès, F. Sotgia, and M. Lisanti, "Chemotherapy induces the cancer-associated fibroblast phenotype, activating paracrine Hedgehog-GLI signalling in breast cancer cells," Oncotarget, vol. 6, no. 13, pp. 10728-10745, 2015.
[81] E. Piskounova, M. Agathocleous, M. M. Murphy et al., "Oxidative stress inhibits distant metastasis by human melanoma cells," Nature, vol. 527, no. 7577, pp. 186-191, 2015.

[82] Y. Liu, Q. Li, L. Zhou et al., "Cancer drug resistance: redox resetting renders a way," Oncotarget, vol. 7, no. 27, pp. 4274042761, 2016.

[83] H. Yan, B.-Y. Guo, and S. Zhang, "Cancer-associated fibroblasts attenuate Cisplatin-induced apoptosis in ovarian cancer cells by promoting STAT3 signaling," Biochemical and Biophysical Research Communications, vol. 470, no. 4, pp. 947-954, 2016. 


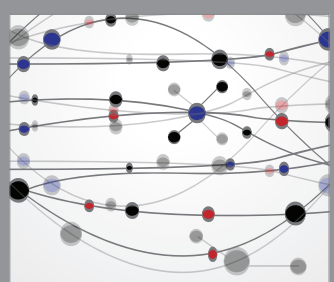

The Scientific World Journal
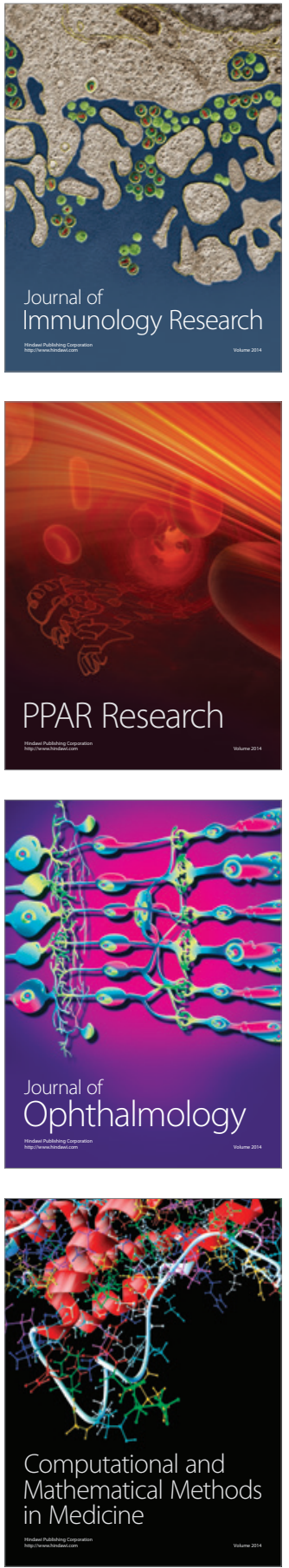

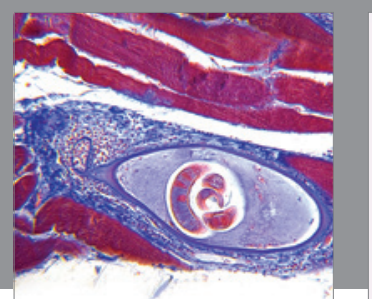

Gastroenterology Research and Practice

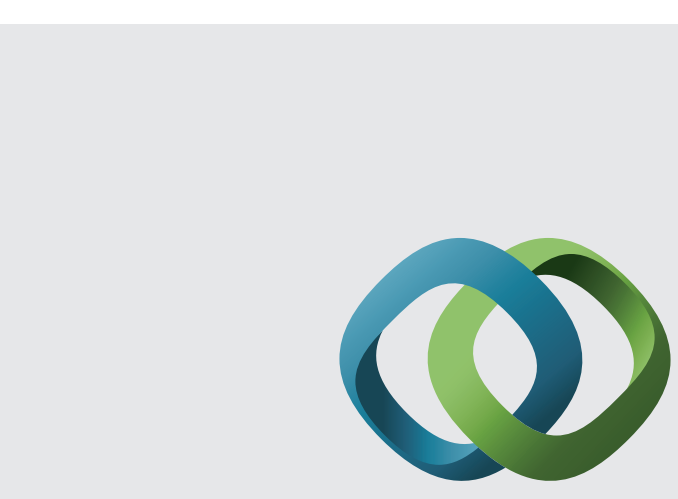

\section{Hindawi}

Submit your manuscripts at

http://www.hindawi.com
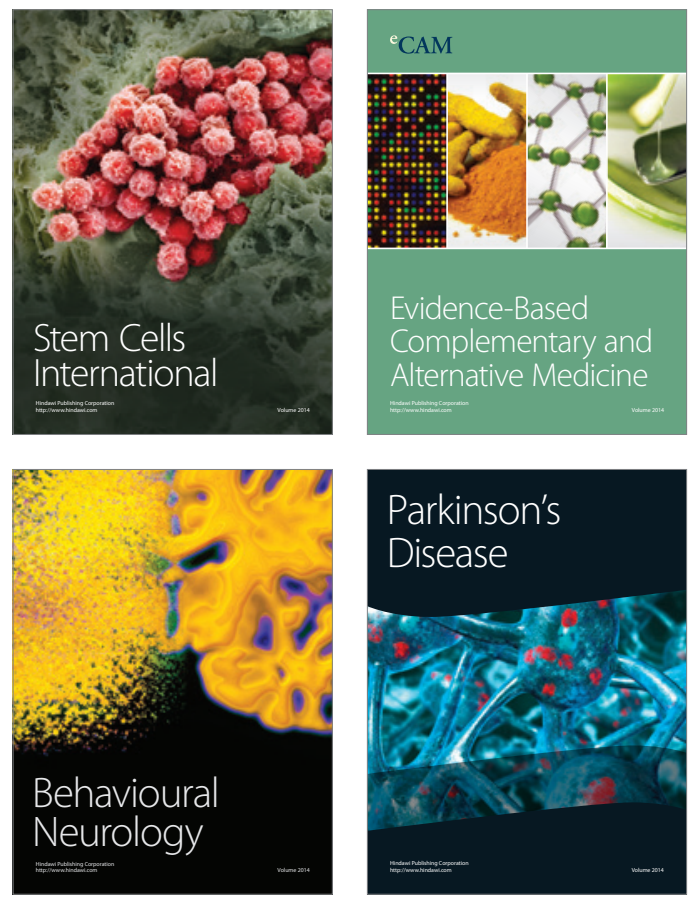
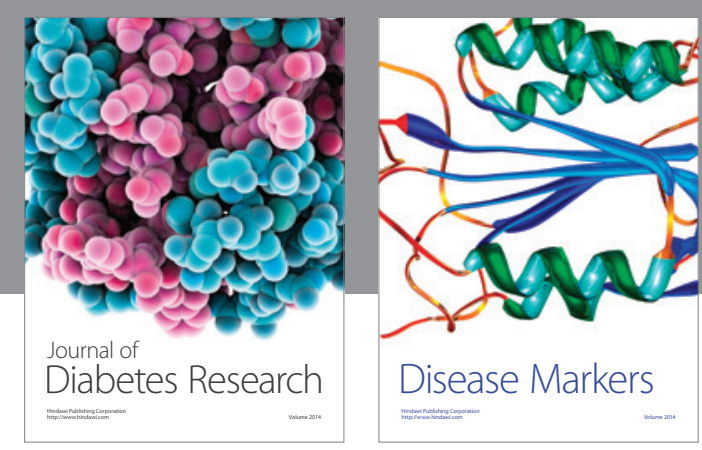

Disease Markers
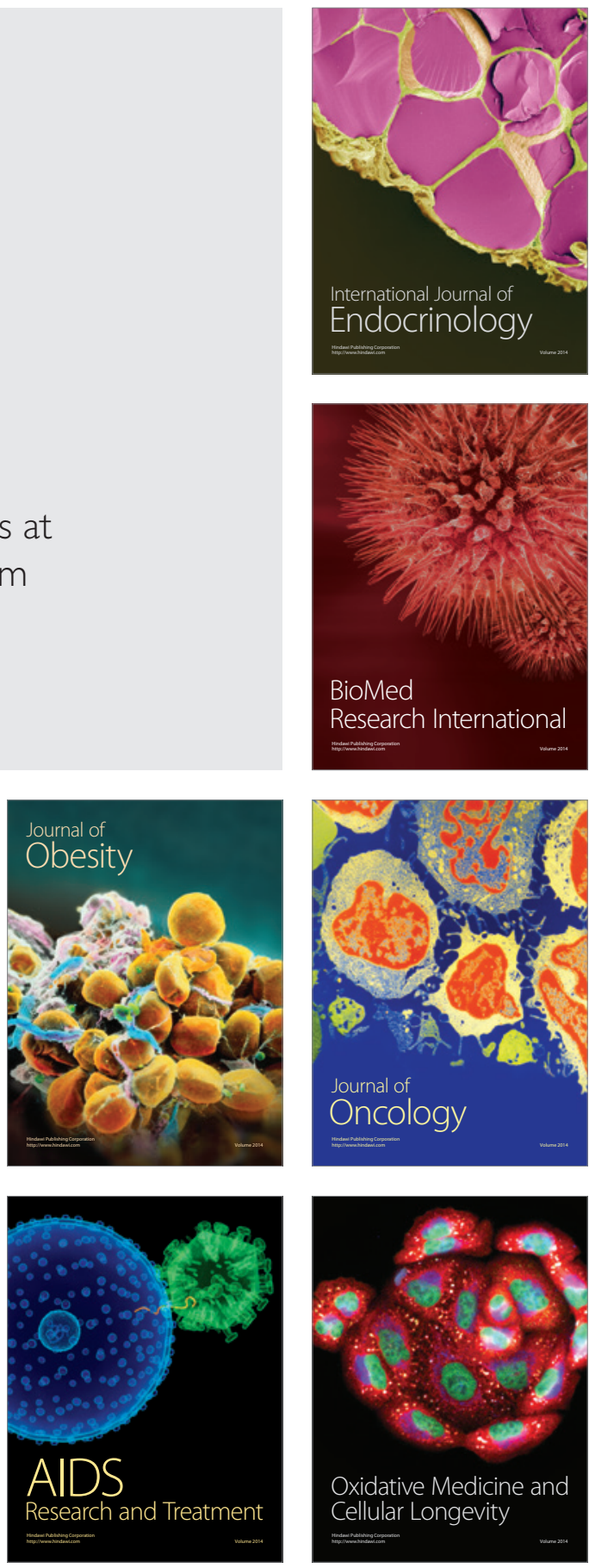